\title{
Six Reasons to Consider Using R in Psychological Research
}

\author{
Tyson S. Barrett ${ }^{1}$ \\ ${ }^{1}$ Utah State University
}

\begin{abstract}
The R Statistical Environment is becoming increasingly accessible to nonstatistician researchers. This provides an opportunity for many researchers in psychology to consider adopting $\mathrm{R}$ in their work. Herein is presented six reasons for why $\mathrm{R}$ should not be overlooked by researchers in psychology: 1) $R$ is free and open-source; 2) $R$ has many packages built for common descriptive and statistical analyses in the psychological sciences; 3 ) the $\mathrm{R}$ community is supportive and have freely provided many useful resources, for beginners and advanced users alike; 4) R is becoming increasingly popular in psychology already, making it easier to collaborate using the software; 5) analyses in $\mathrm{R}$ can be highly reproducible with features like $\mathrm{R}$ Markdown and knitr, simplifying the process to provide open science; and 6) the $\mathrm{R}$ language has improved in diverse ways, making common data wrangling tasks fast, dependable, and highly replicable. These reasons are presented with their corresponding limitations.
\end{abstract}

Keywords: R, Open Science, R Markdown, Reproducibility

Word count: 3261

The "reproducibility crisis" (Collaboration \& others, 2015) has brought to light several avenues for improvement in psychological science (Cumming, 2014; Goodman, Fanelli, \& Ioannidis, 2016; McKiernan et al., 2016; Nosek, Ebersole, DeHaven, \& Mellor, 2018). Arguably, the major theme across recommendations is generally regarding open scienceopen access to data, articles, peer reviews, and code (McKiernan et al., 2016). Before the potential problems with reproducibility became apparent, the Association for Psychological Science began pushing for more training in, and the use of, open-source software, specifically referencing the R Statistical Environment (Blanchar, 2013). In the same vein, the National Institutes of Health in the United States recently launched a new training program to improve quantitative training in the social sciences ("NIH launches new training program

Correspondence concerning this article should be addressed to Tyson S. Barrett, 2800 Old Main Hill, Logan, UT 84322. E-mail: tyson.barrett@usu.edu 
to enhance quantitative training in behavioral and social sciences research," 2019), citing the lack of changes to methodological training for several decades. These recommendations flow seamlessly with the challenge that psychological researchers face in trying to reduce the problems of the reproducibility crisis (McKiernan et al., 2016).

Currently, proprietory software like IBM's SPSS can be preferred in psychological research because of its point-and-click interface, its familiarity with other common software (e.g., Microsoft's Excel), and its ease of use for common methods (e.g., t-test, ANOVA). But some notable problems can occur with the common proprietory software. First, point-andclick analyses can be difficult to reproduce and can remove the researcher from the actual methodologies being employed. Although there are ways to reproduce the results (e.g., saving the syntax output from SPSS), these steps may not be emphasized in the training and use of the software. Second, proprietory software does not update as regularly and as openly as open-source software. That is, for open-source software, updates can be provided by experts across fields as quickly as they can provide them. When proper testing is in place, this can help the software be both reliable and up-to-date. Third, the cost of licenses can create a serious barrier to low-funded researchers and researchers in developing countries. Even with discounts and grants, the sustainability of low-funded researchers to maintain a license is questionable.

On the other hand, the R Statistical Environment has become increasingly accessible to non-statistician researchers. This provides an opportunity for researchers in psychology to consider adopting $\mathrm{R}$ in their work. Six reasons are presented for why $\mathrm{R}$ is worth consideration. These six reasons are:

1. $\mathrm{R}$ is free and open-source.

2. $\mathrm{R}$ has many packages built for common descriptive and statistical analyses in the psychological sciences.

3. The R community is supportive and have freely provided many useful resources, for beginners and advanced users alike.

4. $\mathrm{R}$ is becoming increasingly popular in psychology already, making it easier to collaborate using the software.

5. Analyses in $\mathrm{R}$ can be highly reproducible with features like $\mathrm{R}$ Markdown and knitr, simplifying the process to provide open science.

6. The $\mathrm{R}$ language has improved in diverse ways, making common data wrangling tasks fast, dependable, and highly replicable.

These are presented below, with their corresponding limitations. Before explaining each in more depth, it is important to note that all analyses and examples were completed in $\mathrm{R}$ version 3.5.2 (R Core Team, 2018) using RStudio version 1.2.1114 (RStudio Team, 2018). In fact, all writing, editing, and formatting were done within RStudio, using rmarkdown and papaja R packages (Aust \& Barth, 2018; Xie et al., 2018a). The R Markdown file and data are available at osf.io/436d7. 


\section{$R$ is Free and Open-Source}

According to the $\mathrm{R}$ Foundation, " $\mathrm{R}$ is a free software environment for statistical computing and graphics" with "facilities for data manipulation" (Foundation, 2019). It is provided by the R Foundation at r-project.org, downloadable around the world. Having it available for free is particlarly beneficial to students, early-career researchers, low-funded researchers, and researchers in developing countries. Further, it is used in both academia and industry (e.g., healthcare, government, finance, retail) and has been increasing in popularity (Robinson, 2017). ${ }^{1}$

$\mathrm{R}$ has two main avenues to be updated and/or extended. The first is internal, with the $\mathrm{R}$ Core Team making, often subtle, adjustments to base $\mathrm{R}$. The team works hard to improve the software without those changes "breaking" existing code. These updates result in version increases in $\mathrm{R}$ (e.g., from 3.5.2 to 3.5.3). The second avenue is through the development of add-on packages. Anyone with expertise in $\mathrm{R}$ can create and make these add-on packages available to others. This way of extending $\mathrm{R}$ has been largely beneficial to researchers in psychology and will be discussed next.

\section{Common Descriptive and Statistical Analyses}

As of the analyses of this report, 14788 packages exist on the main package repository, known as CRAN (Comprehensive R Archive Network). To have a package accepted into CRAN it must pass a number of tests and is reviewed by a team of CRAN maintainers. These packages cover topics across the data analytic and statistical spectrum. As such, it is always possible that one of the packages does something similar to what is needed for any given project.

However, 14788 packages is too many to navigate without strategies to find to the most useful and reliable packages. Three common approaches to better navigate the $\mathrm{R}$ package landscape are presented here. First, use the CRAN Taks Views (Zeileis, 2005)collections of packages that perform similar functions. These are maintained by $\mathrm{R}$ experts that occasionally update the lists to show some of the best packages in an area. Three are specifically of interest for psychology: the psychometrics, clinical trials, and social science task views. Second, there are developer groups that have, over time, created groups of packages that have similar syntax and functionality. These are often very well tested. An example is the RStudio developer group, which provide many packages that do various data and statistical work in a unified format (often referred to as the Tidyverse group of packages). Third, it is often beneficial to use the packages that others are using. Colleagues, list-serves, and even social media can often provide recommendations that work well.

With these strategies in mind, three packages that may be useful for descriptive analyses for a broad range of psychological researchers are presented. ${ }^{2}$ These packages, along with helpful functions, and their output are shown in Figure 1. These provide descriptive

\footnotetext{
${ }^{1}$ This indirectly highlights the marketability of being able to use $\mathrm{R}$ for students and researchers looking for careers working with data.

${ }^{2}$ One package, the furniture package, was developed by the author of this article.
} 
statistics, effect sizes, and information about distributions. Although not designed to instruct on what this code means, it makes it clear that it does not take many lines of code to get meaningful information about the variables in a data set using $R$.

There are also many functions and packages that provide statistical methods. $\mathrm{R}$ itself provides functions to do t-tests (t.test ()), the ANOVA family of methods (aov()), and linear modeling $(\operatorname{lm}()$ and $g \operatorname{lm}())$. Other packages provide Bayesian approaches (brms; Bürkner, 2017, 2018), multilevel modeling (lme4; Bates, Mächler, Bolker, \& Walker, 2015), and structural equation modeling (lavaan; Rosseel, 2012), to name only some. ${ }^{3}$ Across a few well-designed packages, it is possible to perform many, if not most, analyses found in psychological journals.

It is also common for methodologists to provide an $\mathrm{R}$ package when they develop a new method, often making $\mathrm{R}$ the only platform to have certain methods. Notably, however, as Wickham (2014) states: "Inconsistency is rife across contributed packages, even within base R. You are confronted with over 20 years of evolution every time you use R." As such, familiarity with each package is important as nuances exist in syntax across packages.

\section{The R Community}

One of the major reasons to start and continue using $\mathrm{R}$ is the $\mathrm{R}$ community. The community is diverse in terms of expertise, identity, location, affiliations, and experience. Mair et al. (2015) found that the social aspect of package development was an important aspect of why package authors perform open-source development. "A high amount of interdependence and feedback from others" (pg. 14791) was found to be an important part of the process. As such, many in the $\mathrm{R}$ community are motivated by collaboration and interdependence between researchers.

This can be seen in the various outreach projects, free resources, and inclusive organizations provided by the community. Official organizations have been created to generate opportunities for low-income, minority-status, and other under-represented individuals (e.g., RLadies Global). Furthermore, many resources, including some leading books, are available for free online (Wickham, 2014; Wickham \& Grolemund, 2016; Xie et al., 2018b).

\section{The Use of $\mathbf{R}$ in Psychology}

A barrier to adopting $\mathrm{R}$ may be the perception that few psychological researchers use it. Previous work has shown that the use of $\mathrm{R}$ is growing in ecology (Lai, Lortie, Muenchen, Yang, \& Ma, 2019), in science in general (Bollmann et al., 2017), and in industry (Robinson, 2017). Yet, at the present, little has been quantified regarding the use of $\mathrm{R}$ for research in psychology previous to this article. Herein, data from the Scopus database were analyzed to answer the following: 1) the extent and growth of the cited use of $\mathrm{R}$ in psychology, and 2) the topics (as informed by the keywords) that are being studied while using R. Because academic journals are the primary source of disseminating research for social scientists and psychologists, the number of citations of $\mathrm{R}$ - that is, the "R Core Team" or " $\mathrm{R}$ Development Core Team" - is likely a good proxy for the general use of $\mathrm{R}$ in these

\footnotetext{
${ }^{3}$ For more information on each of these packages, see their corresponding websites and manuals.
} 
fields. In each research question, the findings were compared to the general pattern of published articles in psychology.

To assess the cited use of $\mathrm{R}$ in psychology, we exported article information from the following Scopus database query: ( REF("R Core Team") OR REF("R Development Core Team")) AND (LIMIT-TO(SUBJAREA, "PSYC")). That is, it queried the references of all the articles in Scopus for "R Core Team" or "R Development Core Team" and then limited the search to psychology. The exported file was a .bib file containing all search results. To analyze the citation data, the following $\mathrm{R}$ packages were used: bibliometrix, tidyr, dplyr, sf, janitor, ggraph, and data.table packages (Aria \& Cuccurullo, 2017; Dowle \& Srinivasan, 2019; Firke, 2018; Garnier, 2018; Pebesma, 2018; Wickham, 2019a; Wickham et al., 2019; Wickham \& Henry, 2019). The collection of articles citing $\mathrm{R}$ was compared to the general articles in psychology in Scopus mentioning any use of "statistic." The search included "statistic" to get an estimate of the articles that could have used R given they are running some sort of statistic but elected not to use it or cite it.

Trend in the Use of $\mathbf{R}$. To understand the trend in using and citing $\mathrm{R}$ in psychological research, the number of times $R$ was cited was assessed by year (see Figure 2 ). This was compared descriptively to the general trend of publications in Scopus in psychology scaled to be on the same scale as the articles that cited R. Furthermore, since the analyses were done in July of 2019, the projected number of articles that would cite $\mathrm{R}$ was calculated for the remainder of the year. This projection was based on a simple, linear projection based on the articles already published in 2019 .

Since 2006, 1213 articles in 192 different journals by 4358 unique authors representing 1455 universities and research institutes cited the R Core Team. 2006 was the first year that any Scopus-indexed social science or psychology article had any reference to the $\mathrm{R}$ Core Team (or R Development Core Team). Figure 1 shows how this has grown quickly since 2006, with an average growth of $86.90 \%$ from 2006 to 2018 . This is far faster than the average growth in the number of published papers in psychology (mean growth of $10.70 \%$ from 2006 to 2018). The major jump in growth occurs starting in 2016. From there, the growth to 2018 was $106 \%$.

However, it is important to note, that even in 2018, articles citing $\mathrm{R}$ only made up $2 \%$ of the total articles published in the field. It is projected to grow to $2.87 \%$ in 2019 .

Topics Covered When Using R. The main topics of the articles citing R - as informed by the keywords - were also analyzed, using term frequencies, to assess what subject areas in psychology were common when using R. This, unfortunately, could not be compared to the general publications in psychology given limitations to the public database query functionality of Scopus.

Of the 1213 articles, the 25 most common keywords included "depression", "individual differences", "reading", and "meta-analysis" (see Figure 3). Even among the top 25 keywords, it is clear that the range of topics covered in the articles using $\mathrm{R}$ span much of psychology and is not confined to certain "quantitative" topics.

\footnotetext{
${ }^{4}$ The search in Scopus for the general articles in psychology was: (( statistic ) AND ( LIMIT-TO ( SUBJAREA, "PSYC" )))
} 


\section{Reproducibility}

Another important reason to consider adopting $\mathrm{R}$ is the free tools that are designed to increase reproducibility of research. These tools are built on R Markdown and knitr (Xie, 2015; Xie et al., 2018a). In conjunction with tools like the Open Science Framework (Foster \& Deardorff, 2017), the entirety of the data analysis and reporting can be more transparent in a way not possible with many other tools.

When used well, R Markdown can be used to create tables, figures, and text directly from $\mathrm{R}$ output. This reduces errors associated with manual data-entry, the inclusion of outdated figures and tables, and other changes that occur during the course of a research project that are not updated in a manuscript. $\mathrm{R}$ Markdown can make it so each value in the text, tables, and figures is updated each time the analysis is updated.

Particularly pertinent to psychological science is the developmental package called papaja (Prepare APA Journal Articles; Aust \& Barth, 2018). It uses R Markdown with the APA 6 LaTex formatting document, and as such, prepares a well-formatted PDF document directly from R. An increasing list of published articles have used papaja. In fact, this article was written entirely using papaja.

A barrier to adoption here is that many collaborators do not use R Markdown for their writing. Many use Microsoft Word or Google Docs, taking advantage of comments and track-changes. One way that researchers have worked around this has been to use the ability of R Markdown to produce Word documents, allow their collaborators to make edits and comments, accept changes and fix errors, and then use that text to update the R Markdown document. This, of course, is not a convenient workflow in some cases. Projects are in the works to be able to convert the edited Word document directly back to R Markdown (e.g., Ross, 2019). In general, these approaches are early in development; therefore, researchers should not expect to be able to use these yet.

\section{The R Language}

The $\mathrm{R}$ language has improved in diverse ways, both through improvements to base $\mathrm{R}$ and through innovative packages. Packages such as dplyr and data.table provide methods to import, format, adjust, reshape, and otherwise manipulate data. These can improve the readability, the speed, and the reliability of the code. Guides to these valuable tools are provided openly (Dowle \& Srinivasan, 2019; Wickham, 2019b; Wickham et al., 2019).

For example, in a few lines of code, we can import the data, add a new variable, get descriptive statistics, and reshape the data from wide to long format. In general, each step is fast, relying on code implemented in $\mathrm{C}$ and $\mathrm{C}++$. Furthermore, we can work with the data that we imported in many ways without ever adjusting the master file in any way. This allows researchers to work with the data in R, try methods, make mistakes, and in the end, our original data set is still the same and can be imported again and worked with another way.

library (data.table)

library (dplyr) 


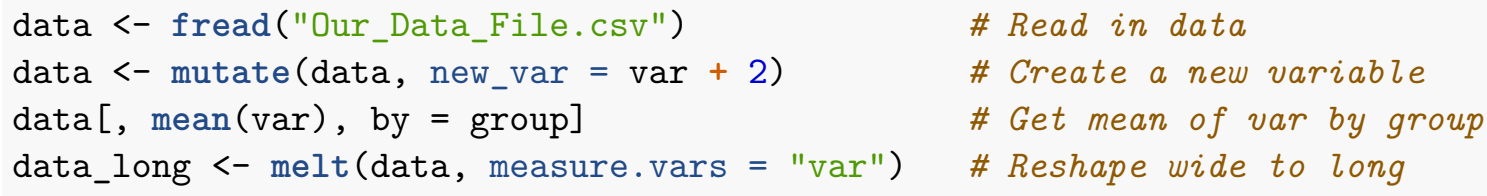

This is in contrast to point-and-click systems that require researchers to save separate data files for each step. This can quickly become difficult to manage, and can be errorprone. Especially problematic for many programs is the last step in the code above, that of reshaping. In spreadsheet programs, this is generally done by copying-and-pasting from different cells to other cells. A few mistakes can lead to serious reliability issues and can mean that the researcher has to start from the beginning, if they noticed the mistake. By using code and a single master file, the analyses can be replicated by anyone with access to both, with the ability to quickly check the reliability of the code.

However, as mentioned earlier, by providing the ability for anyone with expertise to create add-on packages, consistency in syntax style can be lacking. Most researchers that have used several packages in $R$ have seen that nuances of the syntax can vary based on the package. Yet, with only a few packages learned, a researcher can do most data wrangling and analysis necessary. That is, no one needs to learn everything about $\mathrm{R}$ and its packages to be productive.

Another issue encountered by beginners is the inability to interpret the error messages. Work is being done in many packages to improve the information provided in error messages. But, at this point, many error messages can be somewhat unintuitive. With experience, and with the help of the $\mathrm{R}$ community, these error messages can become easier to understand.

\section{Conclusions}

The $\mathrm{R}$ statistical environment is becoming increasingly accessible to non-statistician researchers. Although there are limitations to the software, it is an important option for researchers in psychology to consider using in their research. Not surpringly, then, the use of $\mathrm{R}$ in psychological research appears to be increasing rapidly. This trend may hint at changes to training programs, more comfort in the field with using code, success of outreach from the $\mathrm{R}$ community, the utility of new functionality in $\mathrm{R}$ via add-on packages, and a bigger emphasis on reproducible workflows.

Aria, M., \& Cuccurullo, C. (2017). Bibliometrix: An r-tool for comprehensive science mapping analysis. Journal of Informetrics, 11(4), 959-975. Retrieved from https: //doi.org/10.1016/j.joi.2017.08.007

Aust, F., \& Barth, M. (2018). papaja: Create APA manuscripts with R Markdown. Retrieved from https://github.com/crsh/papaja

Bates, D., Mächler, M., Bolker, B., \& Walker, S. (2015). Fitting linear mixed-effects models using lme4. Journal of Statistical Software, 67(1), 1-48. doi:10.18637/jss.v067.i01 
Blanchar, J. C. (2013). Seven reasons to pursue advanced quantitative training. Observer, 26(10). Retrieved from https://www.psychologicalscience.org/observer/ seven-reasons-to-pursue-advanced-quantitative-training

Bollmann, S., Cook, D., Dumas, J., Fox, J., Josse, J., Keyes, O., ... Debelak., R. (2017). A first survey on the diversity of the $\mathrm{r}$ community. $R$ Journal, 9, 541-552.

Bürkner, P.-C. (2017). brms: An R package for Bayesian multilevel models using Stan. Journal of Statistical Software, 80(1), 1-28. doi:10.18637/jss.v080.i01

Bürkner, P.-C. (2018). Advanced Bayesian multilevel modeling with the R package brms. The $R$ Journal, 10(1), 395-411. doi:10.32614/RJ-2018-017

Collaboration, O. S., \& others. (2015). Estimating the reproducibility of psychological science. Science, $349(6251)$, aac4716.

Cumming, G. (2014). The new statistics: Why and how. Psychological Science, 25(1), $7-29$.

Dowle, M., \& Srinivasan, A. (2019). Data.table: Extension of 'data.frame'. Retrieved from https://CRAN.R-project.org/package=data.table

Firke, S. (2018). Janitor: Simple tools for examining and cleaning dirty data. Retrieved from https://CRAN.R-project.org/package=janitor

Foster, E. D., \& Deardorff, A. (2017). Open Science Framework (OSF). Journal of the Medical Library Association, 105(2). doi:10.5195/jmla.2017.88

Foundation, T. R. (2019). The $r$ project for statistical computing. Retrieved from https: //www.r-project.org

Garnier, S. (2018). Viridis: Default color maps from 'matplotlib'. Retrieved from https: //CRAN.R-project.org/package=viridis

Goodman, S. N., Fanelli, D., \& Ioannidis, J. P. (2016). What does research reproducibility mean? Science Translational Medicine, 8(341), 341ps12-341ps12.

Lai, J., Lortie, C., Muenchen, R., Yang, J., \& Ma, K. (2019). Evaluating the popularity of $\mathrm{r}$ in ecology. Ecosphere, 10.

Mair, P., Hofmann, E., Gruber, K., Hatzinger, R., Zieleis, A., \& Hornik, K. (2015). Motivation, values, and work design as drivers of participation in the $\mathrm{R}$ open source project for statistical computing. Proceedings of the National Academy of Sciences of the United States of America, 112(48), 14788-14792.

McKiernan, E. C., Bourne, P. E., Brown, C. T., Buck, S., Kenall, A., Lin, J., ... others. (2016). Point of view: How open science helps researchers succeed. Elife, 5, e16800.

NIH launches new training program to enhance quantitative training in behavioral and social sciences research. (2019). https://grants.nih.gov/grants/guide/rfa-files/ RFA-OD-19-011.html. 
Nosek, B. A., Ebersole, C. R., DeHaven, A. C., \& Mellor, D. T. (2018). The preregistration revolution. Proceedings of the National Academy of Sciences, 115(11), 2600-2606.

Pebesma, E. (2018). Simple Features for R: Standardized Support for Spatial Vector Data. The $R$ Journal. Retrieved from https://journal.r-project.org/archive/2018/ RJ-2018-009/index.html

R Core Team. (2018). R: A language and environment for statistical computing. Vienna, Austria: R Foundation for Statistical Computing. Retrieved from https://www. R-project.org/

Robinson, D. (2017, October). The impressive growth of r. StackOverflow Blog. Retrieved from https://stackoverflow.blog/2017/10/10/impressive-growth-r/

Ross, N. (2019). Redoc: Reversible reproducible documents. Retrieved from https://github. com/noamross/redoc

Rosseel, Y. (2012). lavaan: An R package for structural equation modeling. Journal of Statistical Software, 48(2), 1-36. Retrieved from http://www.jstatsoft.org/v48/i02/

RStudio Team. (2018). RStudio: Integrated development environment for $r$. Boston, MA: RStudio, Inc. Retrieved from http://www.rstudio.com/

Wickham, H. (2014). Advanced r. Chapman; Hall/CRC.

Wickham, H. (2019a). Forcats: Tools for working with categorical variables (factors). Retrieved from https://CRAN.R-project.org/package=forcats

Wickham, H. (2019b). The tidyverse style guide. Retrieved from https://style.tidyverse.org

Wickham, H., François, R., Henry, L., \& Müller, K. (2019). Dplyr: A grammar of data manipulation. Retrieved from https://CRAN.R-project.org/package=dplyr

Wickham, H., \& Grolemund, G. (2016). $R$ for data science: Import, tidy, transform, visualize, and model data. " O'Reilly Media, Inc.".

Wickham, H., \& Henry, L. (2019). Tidyr: Easily tidy data with 'spread()' and 'gather()' functions. Retrieved from https://CRAN.R-project.org/package=tidyr

Xie, Y. (2015). Dynamic documents with $R$ and knitr (2nd ed.). Boca Raton, Florida: Chapman; Hall/CRC. Retrieved from https://yihui.name/knitr/

Xie, Y., Allaire, J., \& Grolemund, G. (2018a). $R$ markdown: The definitive guide. Boca Raton, Florida: Chapman; Hall/CRC. Retrieved from https://bookdown.org/yihui/ rmarkdown

Xie, Y., Allaire, J. J., \& Grolemund, G. (2018b). $R$ markdown: The definitive guide. Chapman; Hall/CRC.

Zeileis, A. (2005). CRAN task views. $R$ News, 5(1), 39-40. Retrieved from https://CRAN. R-project.org/doc/Rnews/ 


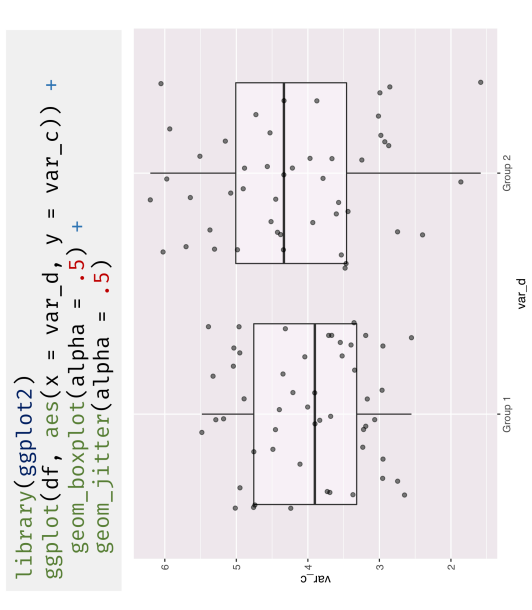

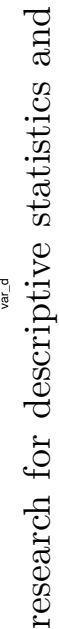

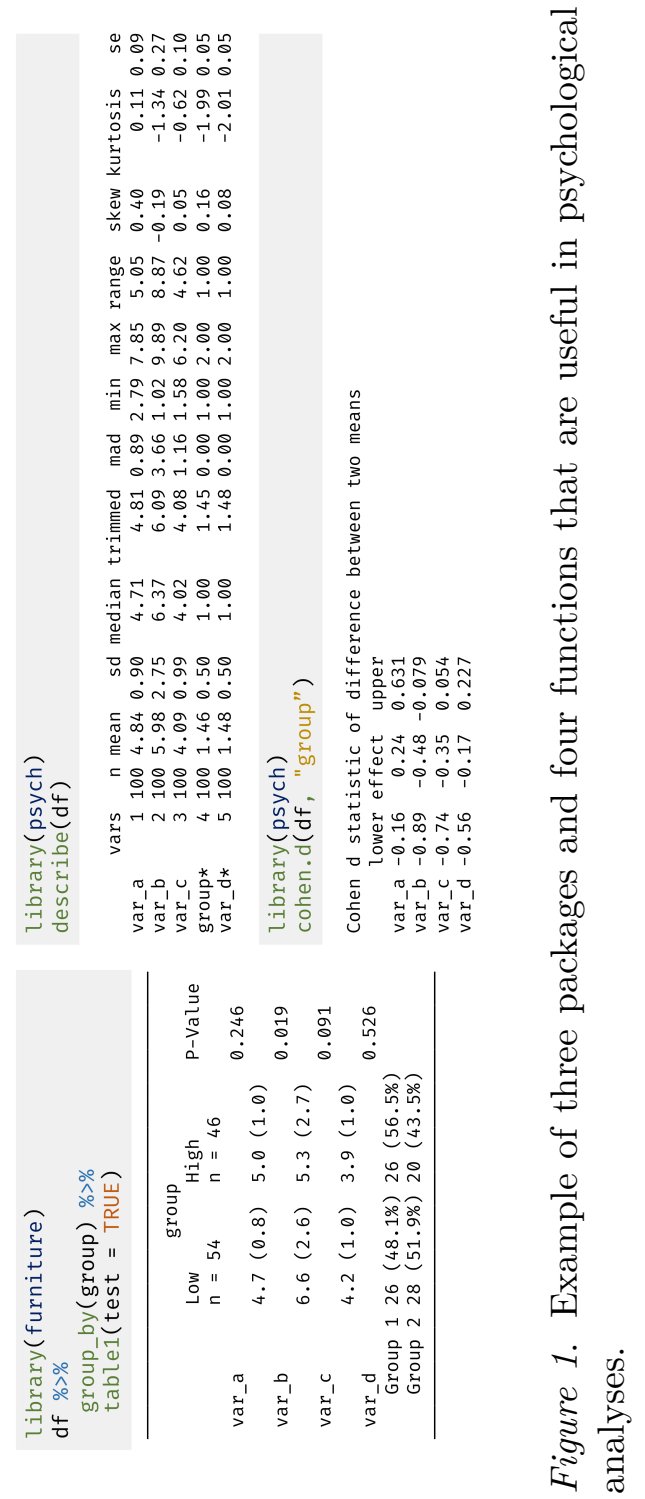




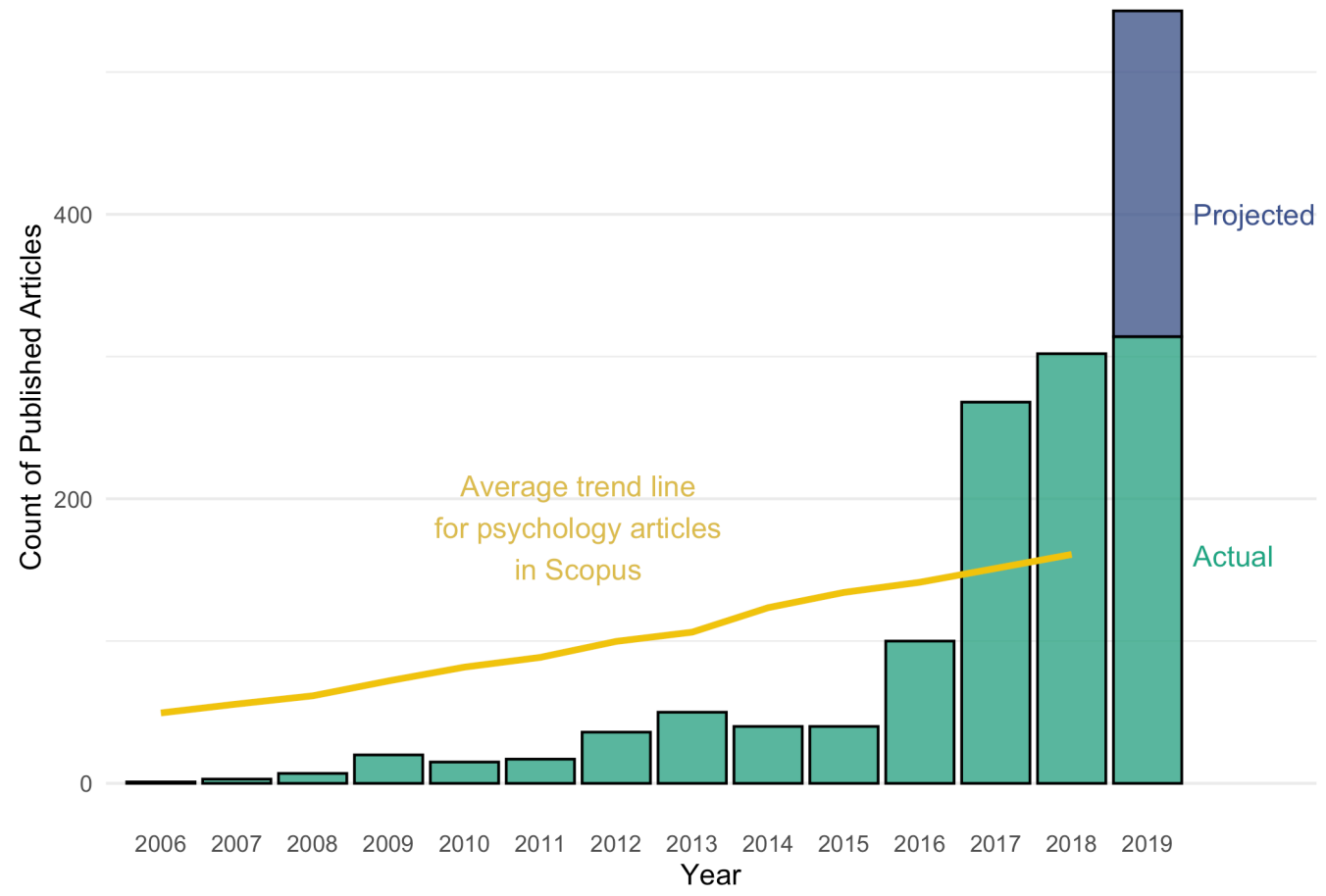

Figure 2. The cited use of $\mathrm{R}$ by year (bars) compared to the average change in the number of publications standardized to the same range as the $\mathrm{R}$ publications (line). Since the analyses were done in July of 2019, the projected number of articles that will cite $\mathrm{R}$ the remainder of the year is also shown. 


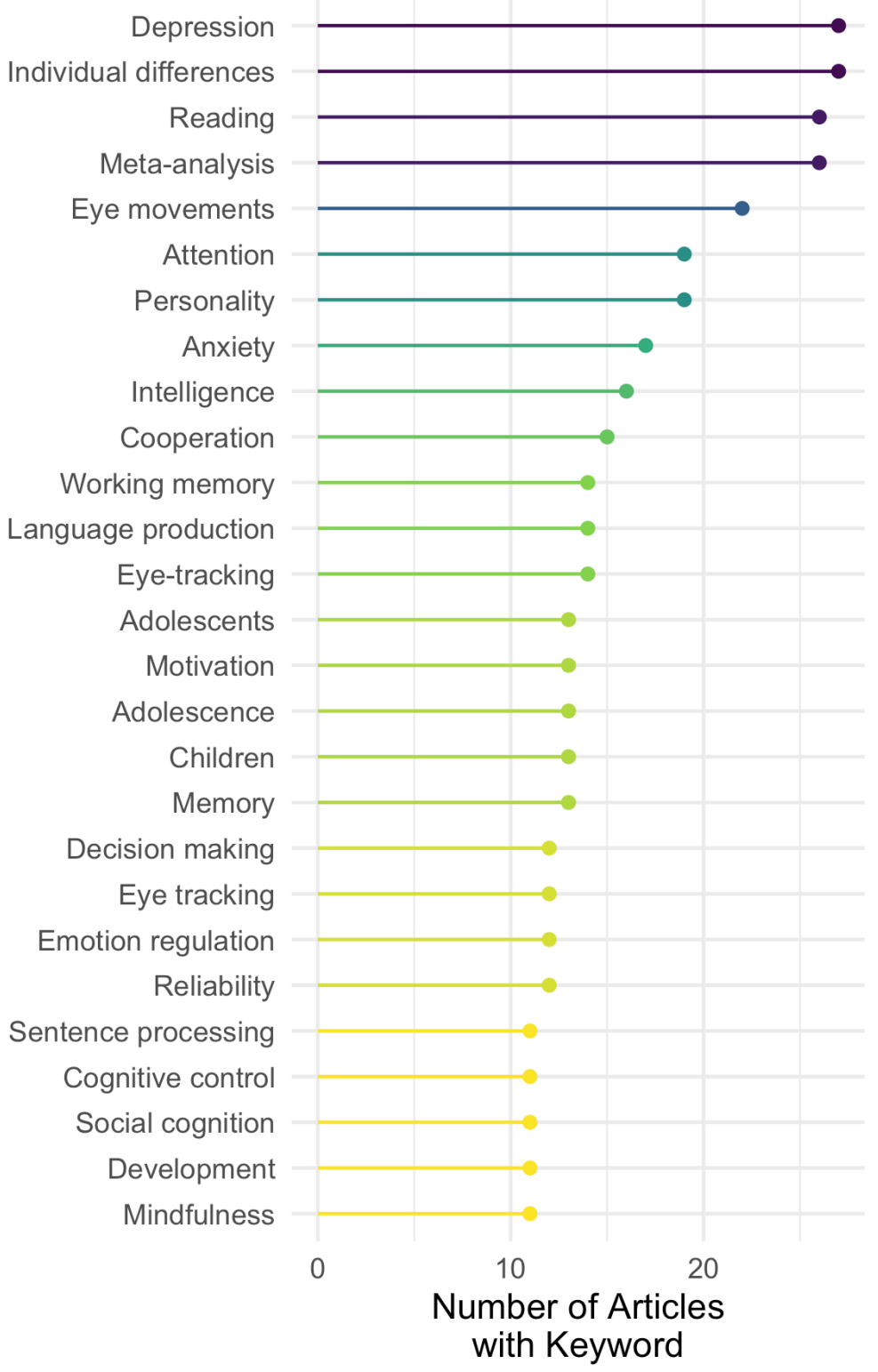

Figure 3. The top 25 keywords in the Scopus-indexed articles that cited R. 